\title{
Seeing obesity as a systems problem
}

\section{Barry Newell ${ }^{\mathrm{A}, \mathrm{C}}$, Katrina Proust ${ }^{\mathrm{A}}$, Robert Dyball ${ }^{\mathrm{A}}$ and Phil McManus ${ }^{\mathrm{B}}$}

AFenner School of Environment and Society, The Australian National University

${ }^{\mathrm{B}}$ School of Geosciences, University of Sydney

${ }^{\mathrm{C} C o r r e s p o n d i n g}$ author. Email: barry.newell@anu.edu.au

\begin{abstract}
Obesity has reached epidemic proportions in many countries and persists despite continuing efforts to find solutions. Such 'stubborn problems' often signal the influence of 'feedback systems'. In the case of the obesity epidemic, this possibility can be investigated using available system analysis tools. The investigation must begin with a study of the interplay between the full range of human and environmental factors. This paper outlines the nature of feedback and briefly discusses some of its management implications. A practical way to initiate a 'systems approach' to the obesity problem is suggested and four principles to guide the management of complex humanenvironment systems are presented.
\end{abstract}

It is likely that the obesity epidemic is a 'systems effect'. That is, there are good reasons to believe that it has emerged from interactions between the variables that characterise human physiology and psychology, and those that characterise urban environments. ${ }^{1,2}$ Interactions of this kind are dominant in such human-urban systems and can give rise to unexpected and unwanted policy outcomes. ${ }^{3}$ In these circumstances it is not useful to look for the main cause of a management problem. Instead it is necessary to develop hypotheses about significant causal links between key system variables. In particular, it is necessary to move beyond thinking in terms of 'causal chains' to develop an ability to see 'causal loops'.

Causal loops are prevalent in complex systems and give rise to 'feedback' behaviour, where the effects of a change act to amplify or oppose the original change. Feedback can generate stubborn management problems. To attack such problems, managers and policy makers must have ways to (a) investigate the causal structure of complex systems, (b) identify possible feedback mechanisms and the behaviours they drive, and (c) identify 'leverage points' where pressure on selected system variables (or the severing of selected causal links) will produce the desired outcomes. These are the elements of a 'systems approach'.

\section{Feedback and its effects}

The 'behaviour' of a human-urban system, the way that its variables change over time, emerges from interactions between these variables. ${ }^{4-6}$ When a change in the value of a system variable is amplified by the system, the effect is called 'positive feedback'. The term 'positive' indicates only that changes are reinforced - it does not mean that the effects are necessarily good. Positive feedback effects are sometimes called runaway effects, bandwagon effects or vicious circles. They can lead to exponential growth or collapse.

When a change is opposed by the system the effect is called 'negative feedback'. Negative feedback effects work to hold system variables at established levels, thus stabilising the system. Note that the term 'negative' indicates only that changes are resisted - it does not mean that the effects are necessarily bad. The various homeostasis mechanisms that maintain stable conditions inside the human body are all examples of 'good' negative feedback.

These effects can be illustrated using 'causal loop diagrams' (the commentary associated with each of the following three figures explains how to approach a causal diagram). Figure 1 provides an example of a systems effect that is commonly called 'policy resistance' - a major cause of stubborn problems. The diagram can be read as follows: a desire for a high level of outdoor activity leads to an increase in the amount of open space preserved in the neighbourhood. Open space increases the popularity of the area. Increased popularity leads to a demand for additional housing. Finally, the demand for new housing leads to development pressures that can result in a reduction in the availability of open space. In this case, a possible leverage point involves breaking one of the links in the feedback loop - for example, the policy resistance can be removed if the pressure to develop open space is resisted.

The example shown in Figure 2 illustrates the amplifying effect of positive feedback. There is a negative feedback loop that operates to maintain the extent of the obesity problem. In this loop an increase in the number of obese individuals causes an increase in the popularity of weightloss programs. Then, the increase in the popularity of weight-loss programs reduces the extent of the obesity problem. But the tendency to see weight-loss programs as 'the solution' reduces efforts to identify and address the 
underlying drivers of obesity. This reduced focus allows urban population and lifestyle factors to increase the power of the drivers, which further increase the number of obese people. Over time the positive feedback loop shifts the balance point maintained by the negative feedback loop. Thus, the overall extent of the obesity problem increases slowly in response to the steady growth of urban populations and the excesses of Western urban cultures.

Complex systems have a large number of 'parts' that interact, via a network of feedback loops, to influence and constrain one another's behaviour. A human-urban system comprises the city's inhabitants plus the ecological, cultural, social and economic entities that make up their environment. Figure 3 shows a portion of the complex human-urban structure that might be expected to drive the obesity epidemic. Even though the diagram illustrates only a part of the possible structure, it is complex enough to show why a systems approach must be adopted when looking for leverage points that can help the community to tackle the epidemic. Note that, if commuting time increases, then the reinforcing loops $\mathrm{R} 1$ and $\mathrm{R} 2$ have the potential to amplify unhealthy behaviours (decreased time spent cooking and exercising). Such 'lock-in effects' are another major source of stubborn problems.

\section{Taking a systems approach}

There are well-developed system analysis tools that can be used to investigate the causal structure of human-environment systems. These range from influence diagrams to sophisticated modelling techniques that allow the construction of working simulations. ${ }^{4,8-10}$ It is possible, however, for an interdisciplinary group to make significant progress working with no more than influence diagrams. Influence diagrams can be thought of as causal loop diagrams (as presented here) without polarities assigned to the arrows. They are easier to produce than causal loop diagrams and so support an unconstrained approach that helps participants to capture their current understanding and intuitions. This freedom also helps to weaken the influence of conceptual barriers between disciplines. ${ }^{11}$

As a first step toward the construction of an influence diagram it is valuable to generate lists of the variables that might be relevant to the target problem. The initial versions of such lists should be compiled in 'brain-storming' sessions involving people from a wide range of disciplines and sectors. See Table 1 for a starting list of variables that are likely to be relevant to the obesity problem. The variables listed include (a) cultural and social factors, (b) the beliefs that constitute community and individual worldviews (mental models), and (c) individual actions that might affect system behaviour.

The lists should be merged and the variables sorted into groups according to their status as (a) system variables, (b) external drivers of the system, and (c) irrelevant to the problem. ${ }^{4}$ Diagrams can then be constructed showing the influence links that are likely to exist between the

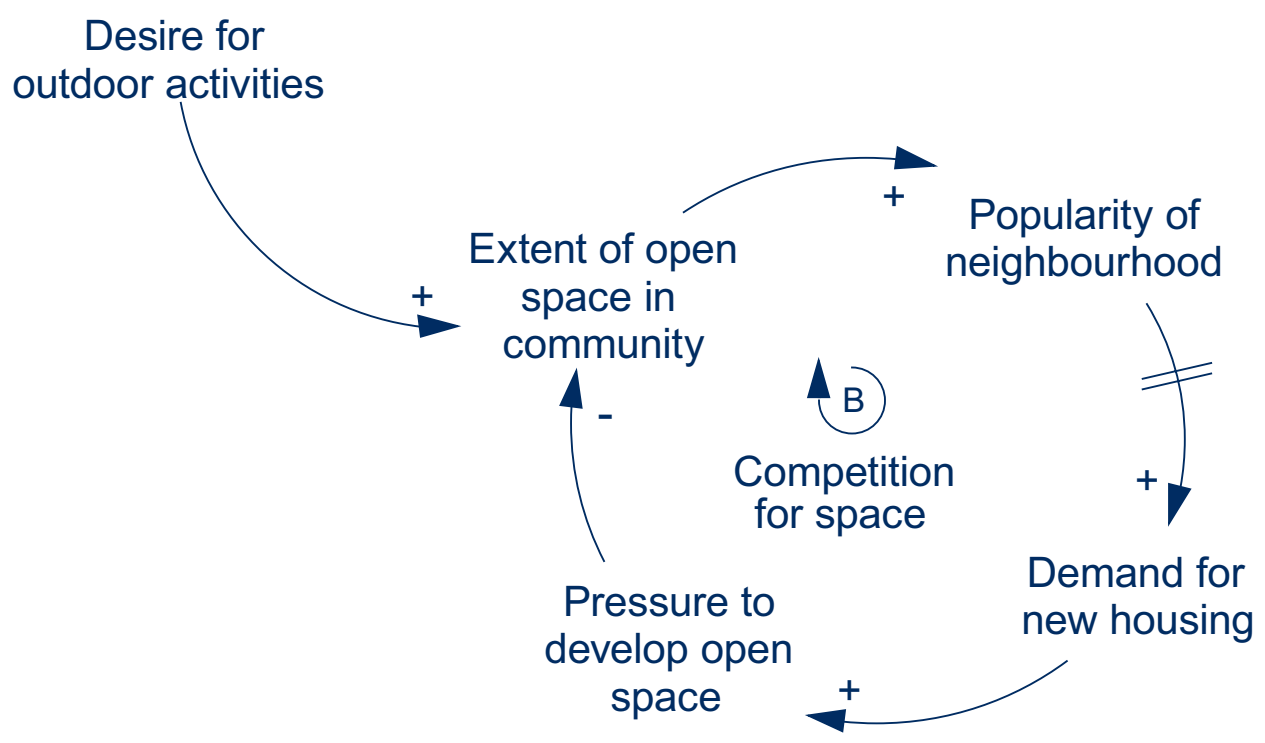

Figure 1. A causal loop diagram illustrating policy resistance.

This 'causal loop diagram' illustrates a negative feedback mechanism that can contribute to obesity by reducing opportunities for physical activity. The blocks of text represent selected system variables. The curved arrows indicate flows of influence between these variables. Each arrow has a symbol that shows its 'polarity.' A plus sign (+) indicates that a change in the variable at the tail of the arrow will cause the variable at the head of the arrow to change in the same direction, assuming that no other causal influences act to cancel out the effect. A minus sign (-) indicates that a change in the variable at the tail of the arrow will cause the variable at the head of the arrow to change in the opposite direction (all else being equal). The short parallel lines drawn across one of the influence arrows indicate a delay in the system. The encircled $B$ in the centre of the feedback loop labelled 'competition for space' indicates that this is a 'balancing' (negative feedback) loop that acts to counteract any change in any of its four variables. 
variables. On this basis the team can then go on to identify possible feedback loops within the system (as was done in Figure 3).

Influence diagrams can provide the starting point for more extensive studies of the dynamics of human-urban systems. Our experience has shown, however, that the process of constructing and discussing influence diagrams can itself help a group to develop a greatly enhanced understanding of the behaviour of their system of interest. Such an endeavour, carried out collaboratively by public-health researchers, policy makers, and providers could provide valuable new insights into the drivers of the obesity epidemic.

\section{Principles for managing complex human-environment systems}

Recent work by Newell and Proust has led to a focus on four principles that can help guide attempts to manage in complex human-environment systems. ${ }^{9,10}$ These principles, expressed in a form appropriate to the obesity problem, can be used to summarise the above discussion:

Principle 1: Feedback effects are important drivers of behaviour in human-urban systems.

Principle 2: Any action taken in a human-urban system will have multiple outcomes, some wanted and some unwanted. The unwanted outcomes will usually be delayed and therefore may go unrecognised.
Principle 3: The behaviour of a human-urban system cannot be optimised by optimising the behaviour of its parts taken in isolation from one another.

Principle 4: The design of effective policies for the management of human-urban systems requires an integrative approach that crosses existing professional and institutional boundaries.

Principles 1 and 2 capture generic aspects of system behaviour as observed in a wide range of situations. The phenomenon of policy resistance, as discussed above, is typical. Principles 3 and 4 capture two characteristics of systems that must be taken in to account in any management intervention. They are intended to counter the natural tendency of managers and policy-makers to focus on subproblems and to institute policies intended to solve each sub-problem separately.

\section{Conclusion}

Feedback system concepts are a natural and necessary component of an integrative approach to the obesity epidemic. Public health policies that are informed by these concepts, and the above system principles, have a good chance of proving effective in the face of this highly stubborn problem.

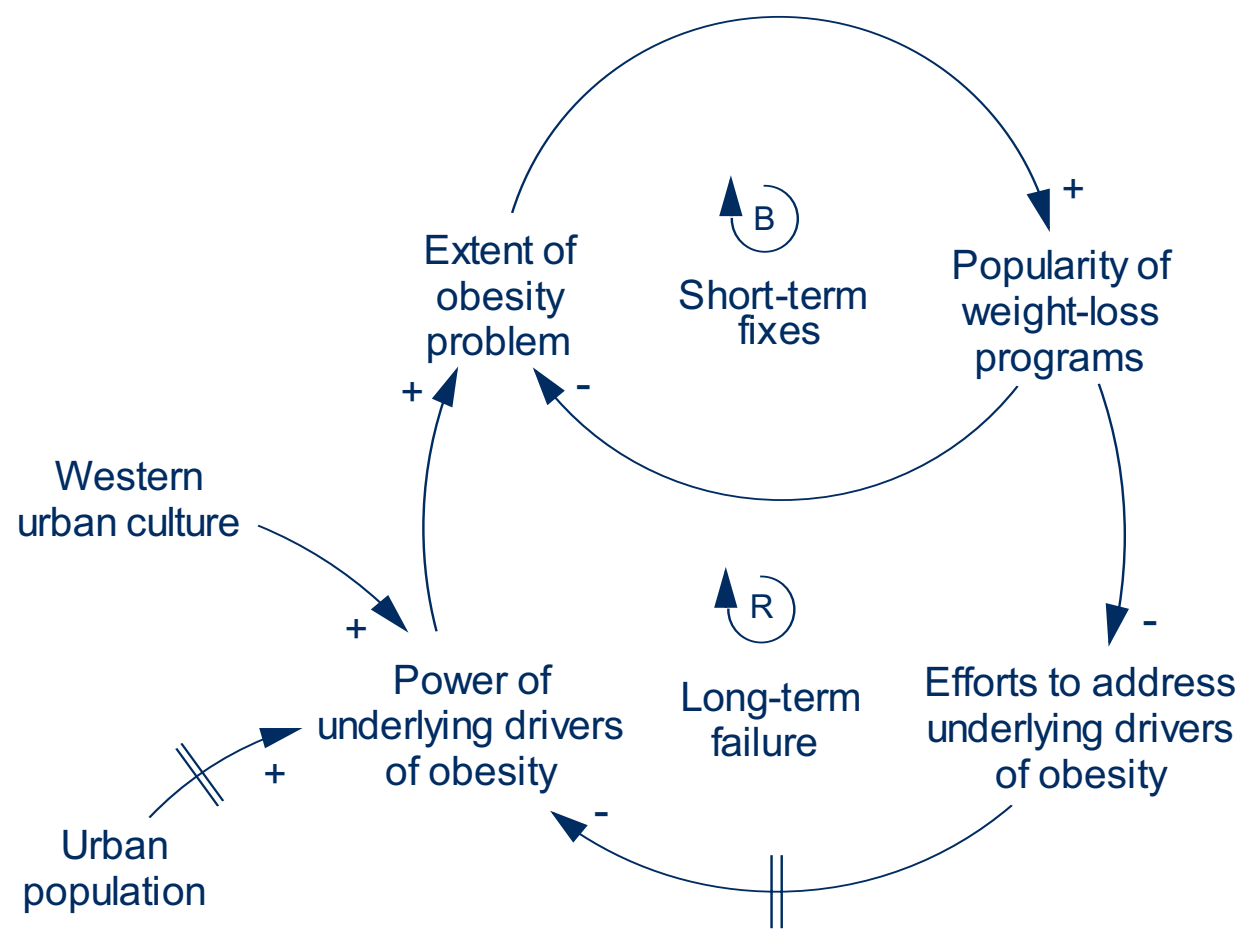

Figure 2. A causal loop diagram illustrating fixes that fail.

This diagram illustrates a commonly occurring situation that can be represented using a causal structure that Senge calls the 'Fixes that Fail' system archetype. ' In this structure a rapidly operating negative feedback loop acts to 'fix' (balance out) the symptoms of a basic problem while a more slowly acting positive feedback loop acts steadily over time to make the basic problem worse. The encircled $R$ in the centre of the feedback loop labelled 'long-term failure' indicates that this is a 'reinforcing' (positive feedback) loop that acts to amplify a change in any of its four variables. 


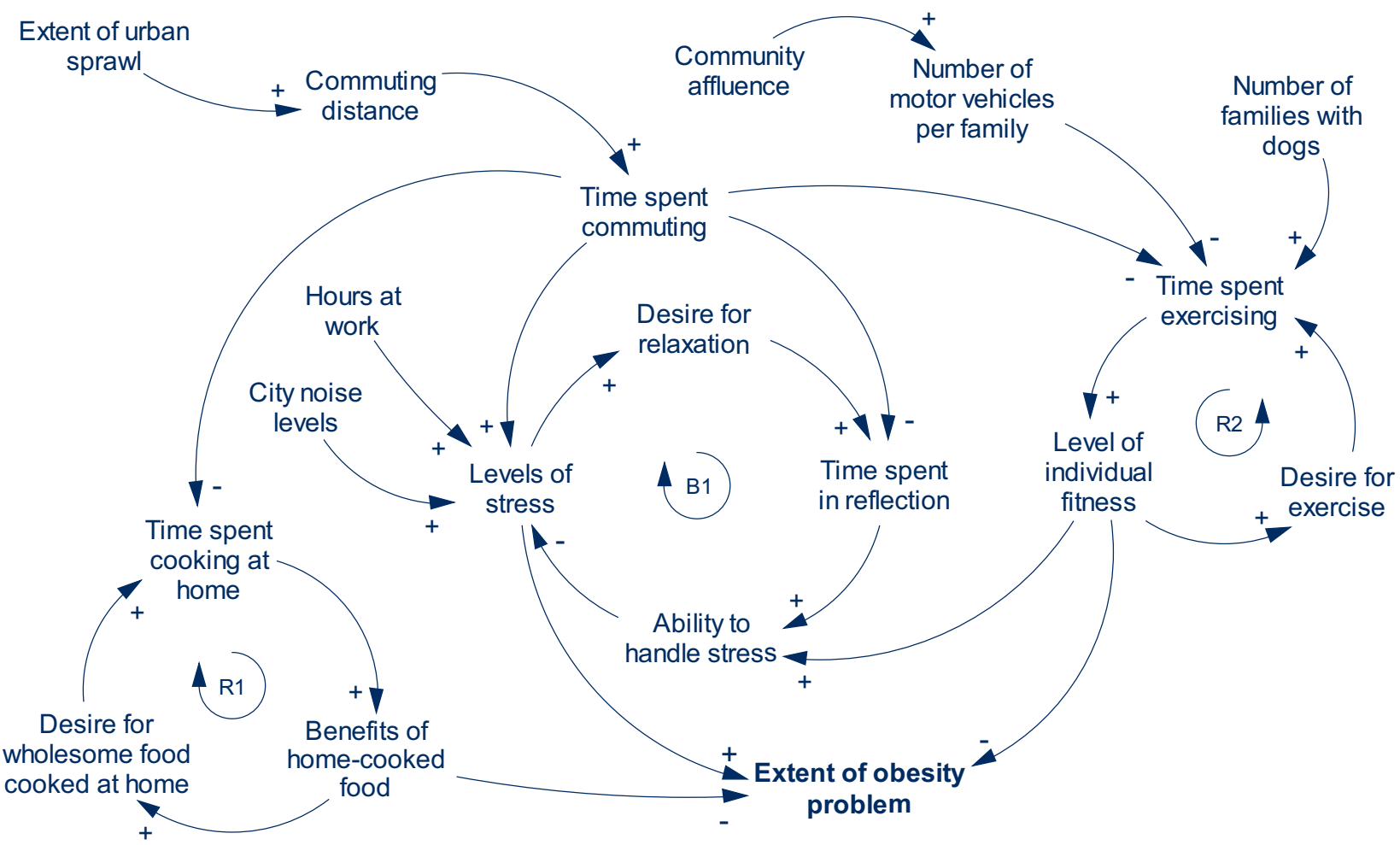

Figure 3. Causal structure proposed for a part of the human-urban system that produces obesity.

This diagram summarises possible systemic effects. To keep the diagram simple a number of influence links have been omitted. For example, the three driving variables shown at the top of the diagram (extent of urban sprawl, community affluence, number of families with dogs) are themselves parts of more extensive feedback loops. There are three feedback loops related to eating (R1), relaxation (B1) and exercise regimes (R2). The variable 'number of families with dogs' is included to stress the need for initial system analyses to include as wide a range of variables as possible.

Table 1. Variables that can affect an individual's body weight

\begin{tabular}{|c|c|c|}
\hline \multicolumn{3}{|c|}{ A. Variables conducive to healthy body weight } \\
\hline Cultural and social & Worldviews & Individual actions \\
\hline $\begin{array}{l}\text { Availability of information about } \\
\text { healthy living }\end{array}$ & Belief in the importance of a healthy lifestyle & $\begin{array}{l}\text { Building in time to achieve a balanced } \\
\text { lifestyle }\end{array}$ \\
\hline Access to healthy food & Understanding of healthy eating regimes & Consuming healthy foods \\
\hline Opportunities for physical activity & $\begin{array}{l}\text { Understanding of the benefits of time } \\
\text { spent exercising }\end{array}$ & Participation in physical activities \\
\hline $\begin{array}{l}\text { Neighbourhood design to support } \\
\text { walking and cycling }\end{array}$ & Belief that outdoor activities are essential & Walking and cycling rather than driving \\
\hline Local employment opportunities & Belief in the value of local employment & $\begin{array}{l}\text { Acceptance of lower-paid work with } \\
\text { reduced travel overheads }\end{array}$ \\
\hline \multicolumn{3}{|c|}{ B. Variables detrimental to healthy body weight } \\
\hline Cultural and social & Worldviews & Individual actions \\
\hline $\begin{array}{l}\text { Time pressure; availability of } \\
\text { labour-saving devices }\end{array}$ & $\begin{array}{l}\text { Belief in competing demands on time; } \\
\text { inclination to save time }\end{array}$ & Buying and using labour-saving devices \\
\hline Population growth; urban sprawl & $\begin{array}{l}\text { Belief that growth is essential and has } \\
\text { no limits }\end{array}$ & $\begin{array}{l}\text { Dependence on motor vehicles; work- } \\
\text { places beyond the local community }\end{array}$ \\
\hline Consumerism & $\begin{array}{l}\text { Belief in market-based commodities and } \\
\text { services, seen as preferable to } \\
\text { self-sufficiency }\end{array}$ & $\begin{array}{l}\text { Succumbing to unhealthy, unnecessary } \\
\text { purchases }\end{array}$ \\
\hline $\begin{array}{l}\text { Inducements to unhealthy } \\
\text { eating traditions }\end{array}$ & $\begin{array}{l}\text { Belief in eating as reward and pastime; } \\
\text { acceptance of fast-food culture }\end{array}$ & $\begin{array}{l}\text { Acceptance of diets high in fat, sugar, salt } \\
\text { and alcohol; consumption of excessive } \\
\text { portions }\end{array}$ \\
\hline $\begin{array}{l}\text { Characteristics of The Entertained } \\
\text { Society }\end{array}$ & $\begin{array}{l}\text { Desire to be entertained; lack of belief in } \\
\text { ability to initiate activities }\end{array}$ & $\begin{array}{l}\text { Excessive TV watching; lack of } \\
\text { imagination and initiative }\end{array}$ \\
\hline
\end{tabular}




\section{Acknowledgements}

We thank Jane Dixon, Debra Summer and a referee for valuable comments.

\section{References}

1. Kearns A, Beaty M, Barnett G. A social-ecological perspective on health in urban environments. NSW Public Health Bull 2007; 18(3-4): 48-50.

2. National Obesity Taskforce. Healthy Weight 2008 -Australia's future. Canberra: National Obesity Taskforce, 2003.

3. Tenner E. Why things bite back. London: Fourth Estate, 1997.

4. Sterman JD. Business dynamics: systems thinking and modeling for a complex world. Boston: Irwin McGraw-Hill, 2000.

5. Richardson GP. Feedback thought in social science and systems theory. Philadelphia: University of Pennsylvania Press, 1991.

6. Jervis R. System effects: complexity in political and social life. Princeton: Princeton University Press, 1997.

7. Senge PM. The fifth discipline: the art and practice of the learning organization. Sydney: Random House, 1992.
8. Newell B, Proust K. The Darwin Harbour Modelling Project: A Report to the Ecological Research Group of the Darwin Harbour Advisory Committee. Darwin: Darwin Harbour Advisory Committee, 2004. At

http://www.nt.gov.au/nreta/water/dhac/publications/pdf/finalre port20050307.pdf, accessed 19 October 2007.

9. Proust K, Newell B. Catchment and community: Towards a management-focused dynamical study of the ACT water system. Canberra: ACTEW Corporation, 2006. At http://www.water.anu.edu.au/pdf/publications/Catchment\%20a nd\%20Community.pdf, accessed 19 October 2007.

10. Proust K, Dovers S, Foran B, Newell B, Steffen W, Troy P. Climate, energy and water: accounting for the links. Canberra: Land \& Water Australia, 2007. At

http://www.lwa.gov.au/downloads/publications_pdf/ER071256. pdf, accessed 19 October 2007.

11. Newell B, Crumley CL, Hassan N, Lambin EF, Pahl-Wostl C, Underdal A et al. A conceptual template for integrative humanenvironment research. Glob Environ Change 2005; 15: 299-307. doi:10.1016/j.gloenvcha.2005.06.003 